\title{
TRIANGULATION OF MANIFOLDS. I
}

\author{
BY R. LASHOF AND M. ROTHENBERG ${ }^{1}$
}

Communicated by William Browder, December 26, 1968.

I. Introduction. The purpose of this note is to outline a proof that almost every compact manifold $M$ of dimension greater or equal to six, (or dimension greater or equal to five if $\partial M=0$ ) can be triangulated as a combinatorial manifold. The word "almost" refers to two types of restrictions we impose on $M$. First that $\pi_{1}(M)$ be sufficiently nice. Secondly, and more unpleasantly we require $H^{4}\left(M ; Z_{2}\right)=0$ and $H^{3}\left(\partial M ; Z_{2}\right)=0$. Since the existence of triangulation implies uniqueness, a consequence of our result is a form of the Hauptvermutung which covers some cases previously not done. For example $\mathrm{Wh}\left(\pi_{1}(M)\right)=0$.

The proofs of the announced theorems make use of a number of recent results, and thus represent a collective mathematical achievement. The fundamental breakthrough is due to Kirby in [3], who first established what we refer to as Lemma 2, conjectured Proposition 3, and recognized its importance for triangulating manifolds and for the annulus conjecture. Other important geometric ingredients are due to Lees [4] and Lashof [5]. A self-contained and straightforward geometric proof of the theorem based on these results is possible if one is willing to assume $M$ is 4-connected. The more general form stated above depends on delicate and difficult algebraic results of Hsiang and Shaneson [2], Shaneson [7], and Wall [8]. We understand that Kirby and Siebenmann also have obtained similar results.

In this note, I, we outline a proof of our main technical result, Theorem 3. In II, we show how this implies the results of the first paragraph.

The following is a useful trick, first observed by Kervaire.

Lemma 1. Let $\phi: X \times R \rightarrow Y \times R$ be a homeomorphism where $X, Y$ are compact spaces and $R$ the real numbers. Then there exist a homeomorphism $\psi: X \times S^{1} \rightarrow Y \times S^{1}$ such that for $\epsilon>0$ and sufficiently small the following diagram commutes:

$$
\begin{aligned}
X & \times[-\epsilon, \epsilon] \stackrel{\phi}{\rightarrow} Y \times R \\
& \downarrow 1 \times \exp \quad \downarrow 1 \times \exp \\
X & \times S^{1} \stackrel{\psi}{\longrightarrow} Y \times S^{1}
\end{aligned}
$$

\footnotetext{
1 Partially supported by an NSF Grant.
} 
Further if $X$ and $Y$ are $P L$ manifolds and $\phi \mid \partial X \times R$ is $P L$ (piecewise linear), then we can choose $\psi$ such that $\psi \mid \partial X \times S^{1}$ is $P L$.

The following lemma is the fundamental geometric observation of Kirby [2].

LEMmA 2. Let $\lambda: B^{k} \times t^{n} \rightarrow B^{k} \times t^{n}$ be a homeomorphism where $B^{k}$ is a homeomorph of the unit $k$ ball in $R^{k}$ and $t^{n}$ is the $n$ torus, the Cartesian product of $S^{1}$ with itself $n$ times. Suppose $\lambda \mid \partial B^{k} \times t^{n}=i d e n t i t y$. Let $\tilde{\lambda}: B^{k} \times R^{n} \rightarrow B^{k} \times R^{n}$ be a covering map of $\lambda$, determined uniquely in case $k \geqq 1$ by the condition that $\tilde{\lambda} \mid \partial B^{k} \times R^{n}=$ identity. Then $\tilde{\lambda}$ is isotopic to the identity by an isotopy that is the identity on the boundary, $\partial B^{k} \times R^{n}$.

Proposition 3. Suppose $k \neq 3$ and $h: B^{k} \times t^{n} \rightarrow B^{k} \times t^{n}$ is a homeomorphism such that $h$ restricted to a neighborhood of $\partial B^{k} \times t^{n}$ is $P L$. Then some finite cover of $h, \tilde{h}$, is homotopic to a PL homeomorphism $h^{\prime}$ with $\tilde{h}=h^{\prime}$ on a neighborhood of $\partial B^{k} \times t^{n}$.

If $k \geqq 5$ then an easy application of the results of Farrell [11] and induction on $n$ shows that $h$ itself is homotopic to an appropriate PL homeomorphism $h^{\prime}$. For $k<5$ the result depends on a delicate analysis of Hsiang and Shaneson [2], or alternatively Wall [8].

Corollary 4. Let $\lambda: B^{k} \times t^{n} \rightarrow B^{k} \times t^{n}$ be a homeomorphism, $k \neq 3$, $P L$ in a neighborhood of $\partial B^{k} \times t^{n}$.

Then some covering map $\tilde{\lambda}: B^{k} \times R^{n} \rightarrow B^{k} \times R^{n}$ of $\lambda$ is isotopic to a $P L$ homeomorphism $\tilde{\lambda}_{1}$ by an isotopy $\tilde{\lambda}_{t}$, with $\tilde{\lambda}_{t}=\tilde{\lambda}$ on a neighborhood of $\partial B^{k} \times R^{n}$.

Proof. First choose a finite cover of $\lambda, \lambda^{1}$ with $\lambda^{1}$ restricted to a neighborhood of $\partial B^{k} \times t^{n}$ extendable to a PL homeomorphism $\alpha$ : $B^{k} \times t^{n} \rightarrow B^{k} \times t^{n}$. This is possible by Lemma 3. Then by Lemma 2 the appropriate universal covering of $\alpha^{-1} \lambda^{1}, \widetilde{\alpha^{-1} \hat{\lambda}^{1}}$, is isotopic via $\gamma_{t}$ to a PL homeomorphism where $\gamma_{t}=$ identity on a neighborhood of $\partial B^{k} \times R^{n}$. Then $\tilde{\alpha} \gamma_{t}$ is an isotopy of $\tilde{\lambda}$ to a PL homeomorphism with $\tilde{\alpha} \gamma_{t}=\tilde{\lambda}$ on a neighborhood of $\partial B^{k} \times R^{n}$.

We now come to our major theorem, which has also been proven independently by Kirby.

THEOREM 1. Let $f: B^{k} \times R^{n} \rightarrow B^{k} \times R^{n}$ be an imbedding of the pair $\left(B^{k} \times R^{n}, \partial B^{k} \times R^{n}\right) \rightarrow\left(B^{k} \times R^{n}, \partial B^{k} \times R^{n}\right)$ such that $f$ maps a neighborhood of $\partial B^{k} \times R^{n}$ piecewise linearly onto a neighborhood of $\partial B^{k} \times R^{n}$. Suppose $k \neq 3, k+n \geqq 5$. Then $f$ is isotopic to a PL imbedding $f_{1}$ through an isotopy $f_{t}\left(f_{0}=f\right)$ such that $f_{t}=f$ on a neighborhood of $\partial B^{k} \times R^{n}$.

Proof. We will prove in detail the weaker result which is sufficient for our purposes, which says that for any $\epsilon>0, f$ restricted to $B^{k} \times D_{\epsilon}^{n}$, 
$D_{\epsilon}^{n}$ is the $n$ disk of radius $\epsilon$, is homotopic through immersions $f_{t}$ to a PL immersion $f_{1}$ where $f_{t}=f$ on a neighborhood of $\partial B^{k} \times R^{n}$. We will indicate how the same method with sufficient attention to the details will imply Theorem 1 as stated.

By the engulfing theorem, there exists an isotopy of the identity $h_{t}: B^{k} \times R^{n} \rightarrow B^{k} \times R^{n}, h_{t}=$ identity on a neighborhood of $\partial B^{k} \times R^{n}$ $\cup f\left(B^{k} \times D_{\epsilon}^{n}\right)$ such that $h_{1} f\left(B^{k} \times R^{n}\right) \supset B^{k} \times D_{r}^{n}$ for any finite $r$.

First choose $r^{\prime}$ with $f\left(B^{k} \times D_{\epsilon}^{n}\right) \subset B^{k} \times D_{r^{\prime}}^{n}$. We can choose a smooth $t^{n-1} \subset R^{n}$ with $0 \in t^{n-1}$ such that $D_{r}^{n,} \subset t^{n-1} \times R \subset R^{n}$. Finally we choose an $D_{r}^{n}$ with $D_{r}^{n} \subset t^{n-1} \times R \subset D_{r}^{n} \subset R^{n}$. We now use the isotopy $h_{t}$ as above to alter $f$, or what is the same thing, we can assume $B^{k} \times D_{r}^{n}$ $C f\left(B^{k} \times R^{n}\right)$.

We then have $B^{k} \times D_{\epsilon}^{n} \subset f^{-1}\left(B^{k} \times D_{r^{\prime}}^{n}\right) \subset f^{-1}\left(B^{k} \times t^{n-1} \times R\right) \subset B^{k} \times R^{n}$. Now as in (6) there exists a PL homeomorphism $i: B^{k} \times t^{n-1} \times R \rightarrow$ $f^{-1}\left(B^{k} \times t^{n-1} \times R\right)$ and thus $f_{0}=f i: B^{k} \times t^{n-1} \times R \rightarrow B^{k} \times t^{n-1} \times R$ is a homeomorphism which is PL on a neighborhood of the boundary. Further $i^{-1}: B^{k} \times D_{\epsilon}^{n} \rightarrow B^{k} \times t^{n-1} \times R$ is PL and by stretching $R$, one can assume that $i^{-1}\left(B^{k} \times D_{\epsilon}^{n}\right) \subset B^{k} \times t^{n-1} \times[-\alpha, \alpha]$, where $\alpha>0$ is small.

We now apply Lemma 1 to get a homeomorphism $\psi$, PL near the boundary, $\psi: B^{k} \times t^{n} \rightarrow B^{k} \times t^{n}$ and a PL embedding $j: B^{k} \times D_{\epsilon}^{n} \rightarrow B^{k} \times t^{n}$ such that $\psi^{\prime} j^{\prime}=f_{0} i^{-1}=f \mid B^{k} \times D_{e}^{n}$. Where $j^{\prime}$ is a lift of $j$ to $B^{k} \times t^{n-1} \times R$ and $\psi^{\prime}$ is a corresponding cover of $\psi$.We now have the following commutative diagram

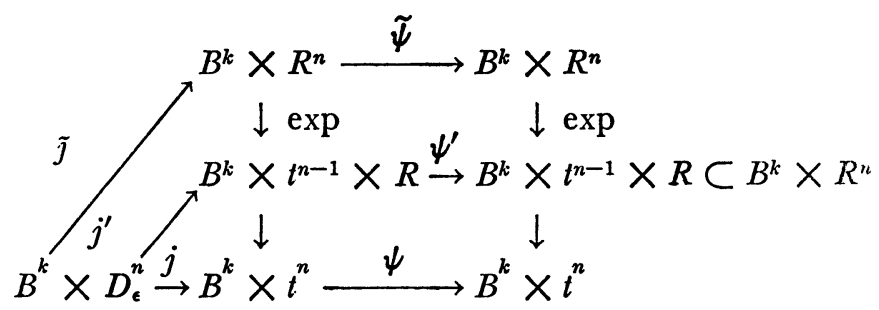

where $\tilde{j}$ is a PL embedding and $f \mid B^{k} \times D_{\epsilon}^{n}=\exp \tilde{\psi} \tilde{j}$. By Corollary 4 , $\tilde{\Psi}$ is isotopic relative to a neighborhood of the boundary to a PL homeomorphism $\tilde{\psi}_{1}$, through isotopy $\tilde{\psi}_{t}$. But then exp $\psi_{t} \tilde{j}$ provides the required homotopy through immersions. This proves the weaker result.

To prove the theorem as stated it is sufficient to show $\tilde{\psi} \tilde{j}$ is isotopic to $\psi^{\prime} j^{\prime}$ through an ambient PL isotopy of the identity on $B^{k} \times R^{n}$. This can be done along the lines of [6]. Q.E.D.

TheOREM 2. Let $n>k, k \neq 3$ and $n \geqq 5$. Then $\pi_{k}\left(P L_{n}\right) \rightarrow \pi_{k}\left(T o p_{n}\right)$ is surjective.

Proof. Let $\left(D^{n}, D^{k}\right)$ be the standard disk pair. Let $\rho: U \rightarrow D^{n}$ be 
a topological immersion, where $U$ is a neighborhood of $D^{k}$ in $D^{n}$, such that $\rho$ restricted to a neighborhood of $\partial D^{k}=$ identity. We identify $\rho, \rho^{\prime}$ if they agree on a neighborhood of $D^{k}$. Isotopies of such (i.e. "regular homotopies") are defined in the obvious way, and we let $I_{\text {Top }}^{n, k}$ be the set of isotopy classes.

By Lees immersion theorem [4], $I_{\mathrm{Top}}^{n, k}=\pi_{k}\left(\mathrm{Top}_{n}\right)$. If we replace topological by piecewise linear in the above, we define $I_{\mathrm{PL}}^{n, k}$, and by the result of Haefliger-Poenaru [1], $I_{\mathrm{PL}}^{n, k}=\pi_{k}\left(\mathrm{PL}_{n}\right)$ and we have a commutative diagram induced by the map $\mathrm{PL}_{n} \rightarrow \mathrm{Top}_{n}$ :

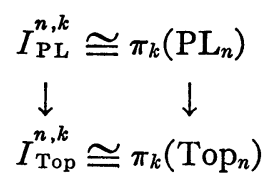

Then the theorem will follow if we can show that every germ of a topological immersion $\rho: U \rightarrow D^{n}, \rho=$ identity in a neighborhood of $\partial D^{n}$, is isotopic to a PL immersion.

We can assume by shrinking $U$, that $U=D^{k} \times D_{\epsilon}^{n-k}$. The immersion $\rho: U \rightarrow D^{n}$ induces a PL-structure on $U$ such that if we denote $U$ with the induced PL-structure by $W, \rho=h \rho^{\prime}$; where $\rho^{\prime}: W \rightarrow D^{n}$ is a PL-immersion and $h: U \rightarrow W$ is a homeomorphism with $h \mid \partial U$ PL.

We consider two cases.

Case I. $k<n / 2, k \neq 3$.

We may extend the PL embedding of $h \mid \partial D^{k}$ to a PL embedding of $\left(D^{k}, \partial D^{k}\right) \rightarrow(W, \partial W)$. Since $k<n / 2, D^{k}$ has a trivial normal PL bundle. By engulfing it follows that $W$ is PL equivalent to $D^{k} \times R^{n-k}$. By Theorem $1, h$ is isotopic to a PL homeomorphism relative to $\partial U$. Hence $\rho$ is isotopic to a PL immersion relative to $\partial U$.

Case II. $k \geqq n / 2, k \neq 3$.

Since $n \geqq 5, k \geqq 4$. Then we may apply Siebenmann's relative splitting theorem [6], to prove $W$ is PL equivalent to $D^{k} \times R^{n-k}$. For the case $k=4$, one must use the fact that any PL manifold, the homotopy type of $S^{4} \times S^{1}$, is PL equivalent to $S^{4} \times S^{1}$ [7]. Apply Theorem 1 to obtain our result.

Addendum. For $k=3$, and $n \geqq 5, \pi_{3}\left(\operatorname{Top}_{n} / \mathrm{PL}_{n}\right)$ is at most $Z_{2}$.

Hsiang and Shaneson have shown the obstruction to the splitting theorem and the suitability of $(k, n)$ for $k=3$, lies in $Z_{2}$. Further, our construction defines a homeomorphism $\lambda$ of $\pi_{3}\left(\mathrm{Top}_{n} / \mathrm{PL}_{n}\right)$ into $Z_{2}$. Since $\alpha \in \pi_{3}\left(\mathrm{Top}_{n} / \mathrm{PL}_{n}\right)$ vanishes if and only if the obstruction $\lambda(\alpha)$ vanishes, $\lambda$ is a monomorphism.

THEOREM 3. For $n \geqq 5, k<n, k \neq 3: \pi_{k}\left(P L_{n}\right)=\pi_{k}\left(\right.$ Top $\left._{n}\right) .^{2}$

2 In the stable case, $n=\infty$, this was first proved by Kirby and Siebenmann. 
PRoof. For $k<n-1$, this follows from Theorem 2, and the fact that $\pi_{k}\left(\mathrm{PL}_{n}\right) \rightarrow \pi_{k}(\mathrm{PL})$ and $\pi_{k}(\mathrm{PL}) \rightarrow \pi_{k}$ (Top) are monomorphisms (see $[6]$ and $[10])$.

For $k=n-1$, consider the commutative diagram:

$$
\begin{aligned}
& \pi_{n}\left(\mathrm{PL}_{n+1}\right) \rightarrow \pi_{n}\left(\mathrm{PL}_{n+1}, \mathrm{PL}_{n}\right) \rightarrow \pi_{n-1}\left(\mathrm{PL}_{n}\right) \rightarrow \pi_{n-1}\left(\mathrm{PL}_{n+1}\right) \\
& \downarrow \alpha_{1} \quad \downarrow \alpha_{2} \quad \downarrow \alpha_{3} \quad \downarrow \alpha_{4} \\
& \pi_{n}\left(\operatorname{Top}_{n+1}\right) \rightarrow \pi_{n}\left(\operatorname{Top}_{n+1}, \operatorname{Top}_{n}\right) \rightarrow \pi_{n-1}\left(\operatorname{Top}_{n}\right) \rightarrow \pi_{n-1}\left(\operatorname{Top}_{n+1}\right)
\end{aligned}
$$

By the first paragraph of this proof, $\alpha_{4}$ is an isomorphism. By (1) of [10], $\pi_{n-1}\left(\mathrm{PL}_{n+1}, \mathrm{PL}_{n}\right)=0$; and by (12) of [10], $\alpha_{2}$ is a monomorphism, $\alpha_{3}, \alpha_{1}$ are onto by Theorem 2. It follows by diagram chasing that $\alpha_{3}$ is an isomorphism.

REMARK. Since $\alpha_{3}$ is an isomorphism all $n \geqq 5, \alpha_{1}$ is an isomorphism, and hence $\alpha_{2}: \pi_{n}\left(\mathrm{PL}_{n+1}, \mathrm{PL}_{n}\right) \rightarrow \pi_{n}\left(\mathrm{Top}_{n+1}, \mathrm{Top}_{n}\right)$ is an isomorphism all $n \geqq 5$.

REMark. If we denote by $\mathrm{PL}_{n}^{\sim}$, Top $\tilde{n}$ the corresponding block bundle groups, Theorem 1 may be interpreted as stating that $\pi_{k}\left(\mathrm{PL}_{n}^{\tilde{n}}\right)=\pi_{k}\left(\mathrm{Top}_{n}\right)$ all $n, k \neq 3$. Also $\pi_{k}\left(\operatorname{Top}_{n} \mid \mathrm{PL}_{n}^{\tilde{n}}\right)=\pi_{k}(\mathrm{Top} \mid \mathrm{PL})$ all $k, n \geqq 5$.

\section{BIBLIOGRAPHY}

1. A. Haefliger and V. Poenaru, La classification des immersions co-combinatoires, Inst. Hautes Etudes Sci. Publ. Math. No. 23, 1964, pp. 75-91.

2. W. C. Hsiang and J. L. Shaneson, Fake tori and the annulus conjecture, Mimeographed Notes, Yale University, New Haven, Conn., 1968.

3. R. C. Kirby, Stable homeomorphisms, Mimeographed Notes, Institute for Advanced Study, Princeton, N. J., 1968.

4. J.Lees, Immersions and surgeries on topological manifolds, Mimeographed Notes, Rice University, Houston, Tex., 1968.

5. R. Lashof, Lees' immersion theorem and the triangulation of manifolds, Mimeographed Notes, University of Chicago, Chicago, Ill., 1968.

6. R. Lashof and M. Rothenberg, Hauptvermutung for manifolds, Conference on the Topology of Manifolds, Complementary Series in Mathematics, Prindle, Weber and Schmidt, Boston, Mass., 1968, pp. 81-105.

7. J. Shaneson, Embeddings with codimension two of spheres in spheres, Bull. Amer. Math. Soc. 74 (1968), 972-974.

8. C. T. C. Wall, verbal report.

9. L. Siebenmann, The obstruction to finding a boundary for an open manifold of dimension greater than five, Thesis, Princeton University, Princeton, N. J., 1965.

10. M. Hirsch, On tubular neighborhoods, Conference on the Topology of Manifolds, Prindle, Weber, and Schmidt, Boston, Mass., 1968, pp. 63-80.

11. F. T. Farrell, Ph.D. Thesis, Yale University, New Haven, Conn., 1967.

12. C. T. C. Wall, Surgery on compact manifolds, Mimeographed Notes, Liverpool, 1967.

University of Chicago, Chicago, Illinois 60637 